\title{
Research on the Translation Strategy of Culture-Load Words in Internal Classic Plain Questions
}

\author{
Shuang Wang ${ }^{1, \text { a }}$ \\ ${ }^{1}$ First English Department, Qiqihar Medical University, Qiqihar, Heilongjiang, 161006 \\ aemail,
}

Keywords: Internal Classic. Plain Questions, Culture-Loaded Words, English Translation

\begin{abstract}
Yellow Emperor Internal Classic" is also called "Nei Jing" and establishes the basic framework for the system of traditional Chinese medicine theory. It plays a decisive role in the Chinese classics. "Nei Jing" takes use of refined language, reflecting the rich cultural flavor, its value and the value of the medical literature are particularly high. "Nei Jing" includes "Q" and "coffin" in the form of questions and answers of the Yellow Emperor and courtiers written. "Nei Jing" load word particularly, highlights of Chinese culture and traditions, is translating "Nei Jing" difficult and important, it has a direct impact on the quality of the translation quality translation. In translating "Internal Classic Plain Questions ", we should select the method to the correctly translate English of culture-loaded words and gradually improve their English translation effects.
\end{abstract}

\section{Introduction}

TCM occupy an important position in China's traditional culture, the protection of people's health in the thousands of years of history. The rapid development of today's medicine, Chinese medicine is still in treatment, prevention field reflects the important role that many Westerners have come to understand Chinese medicine and amazing magical effect [1]. TCM classics "Yellow Emperor" is the oldest, reflecting the ancient medical achievements, and lays a solid foundation for the development of Chinese medicine. However, because of this medical literature it has a strong professional, coupled with its syntactic structure, characteristic words, and there are significant differences in modern Chinese, its esoteric, obstacles and bring influence to spread traditional Chinese medicine theory, its cultural load word translation is "Yellow Emperor" key, so culture-loaded word translation accuracy is particularly critical.

\section{The Culture-Loaded Words of Characteristics and Classification}

Culture - loaded Words primitive cultural information contains words, it is difficult to find the corresponding words in the translation language. These words reflect a nation established in the course of historical development, there are significant differences with other ethnic lifestyle.

The Characteristics of Culture-Loaded Words. First, the culture-loaded words have academic characteristics [2]. TCM English in the field of science and technology in English, does not belong to the category of literary translation, science and technology English more scientific, rigorous, requiring translators to deal with the Chinese culture to improve the wording of accuracy when loaded Words. Secondly, the culture-loaded words have special characteristics. Most TCM culture-loaded words reflects the culture and traditional Chinese medicine theory of traditional Chinese medicine, Chinese medicine is the Five Elements such as terminology, in translation in maintaining its qualities to the maximum extent. Finally, load the word with simple cultural characteristics. TCM terms are highly condensed language translation Loaded Words To concise. There are a lot of books because the Chinese term translated in different countries vary, impact TCM dissemination and exchange in the world, the need to develop international standards to manage. Currently, it is promulgated by the World Association of Chinese medicine "Chinese bilingual basic terminology standards" and "traditional medicine standard nomenclature" issued by the World Health Organization standard for comparison. Translation of TCM culture-loaded words, we should try to choose the interpretation, transliteration, etc., to better highlight the features of our 
culture, promoting the development of Chinese culture in the world.

The Classification of Culture-Loaded Words. "Internal Classic. Plain Questions" Culture loaded Words include the following categories:

Names: such as Fearless, disambiguation, the Yellow Emperor, etc., "Yellow Emperor" word was used transliteration directly translated into "Yellow Emperor", translation was intended to be translated into "Yellow Emperor";

Place name: such as Liuhe, Kyushu, most with transliteration translated into "liuhe", "jiuzhou";

Solar terms, the climate categories: such as the eight century, long summer, twelve, etc. "Long summer" do not mean the summer, but this time from the beginning of autumn to the autumnal equinox, which is unique in the way people behave in ancient time, to understand the ancient Chinese culture needs to be translated "Yellow Emperor • Q" when. "Long summer" was translated into "late summer", "long summer", "last summer", on Chinese medicine perspective, the final translation is more appropriate.

The term class: is a special term acupuncture and Chinese medicine terms, such as lunar, Yang Ming, Ren, Tin Kwai, this word because it is unique to Chinese medicine, it should be directly translated.

Organs, organ categories: direct use and human organs corresponding terminology translation of human organs, such as the "kidney" is the "kidney" "Heart" is the "heart" and so on, this translation method can make translations simpler, different cultures more good communication and integration.

\section{Internal Classic. Plain Questions Translating Approaches to Cultural Loaded Words}

When translating "Internal Classic. Plain Questions" Culture - loaded words, according to the translation of different purposes, to comply with "loyalty" and "function" principle, considering the original text, translators, readers and other factors [3], choose a different translation skills and translation methods to achieve the desired functionality Zevin achieve spread, carry forward the Chinese culture purposes.

Literal Translation. This method is maintained in its original form and content translation method, "Internal Classic. Plain Questions" translation process and can choose the English vocabulary and vocabulary corresponding to the Translation of TCM way. Such as "interior deficiency" (in the virtual), "stomach cold" (stomach), "stomach heat" (stomach), "clearing lung fire" (Qingfei fire), "clearing hear fine" (pure heart and fire) and the like.

Literally Filling Method. Literal translation is possible to maintain the "Yellow Emperor • Q" in its original color and style, deep content, surface sentence are relatively fit when using a literal translation is the best choice. But some words reflect the national culture color, it will lead to literal language readers appeared puzzled and confused. The Select literally filling method can improve the sharpness of the target readers, in order to better carry forward the Chinese culture. Such as Chinese medicine within the meaning of fine, gas, god Sambo, black, white, red, yellow, green is colored, can be translated as "five colours, referring to the colours of green red yellow white and black, which match the five zang organs. " Using this method allows the translation of the translation is more user-friendly.

Transliteration. This method is to use Wade formula or Pinyin phonetic translation. Translating "Internal Classic. Plain Questions" higher proportion of transliteration used. If there is no corresponding vocabulary and Chinese medicine, "qi" in the English, before it was translated into the delivery of "vital energy", "energy" only reflect the "gas" deemed to be "dynamic" meaning, not fully demonstrate all the connotations of "Qi". Choose transliteration can solve this problem, the "gas" translated as "qi", to maintain the original meaning, the reader misunderstand the situation will not occur, this method is easily understood and accepted by foreign readers.

Filling Method Transliteration. If only the reader can not understand the translation transliteration, in order to achieve communication, communication, and maintain the cultural connotation of Chinese medicine, you can choose to raise transliteration method. Such as "wind off" Chinese term meaning encompasses three aspects: a pediatric diagnosis region fingerprints; massage the site name; by name outside the points. Partial translations for the "wind pass", if the 
recipient and users understand the three meanings of the word, or for the realization of simple, brief purpose can be translated; if the recipient of the translation is difficult to understand the word, we must choose transliteration filling method detailed translating "wind off" three meanings. Translation of Chinese names can also use the same method, such as Pinellia can be translated as "banxia (pinellia tuber)".

Increase Translation. "Yellow Emperor" has a lot of culture-loaded words, this is a classical Chinese medical books written, reflecting the concise features. When the translation is to be understood, understanding the meaning of the source language, and then translated, the other to improve the accuracy of the original, to facilitate understanding and learning. Such as the "Q" Ling Lan secret code theory "the lung, phase Fu officer, governance section of the Yan." Original lung compared to "the phase Fu officer" used the metaphor of the way; and the translation is "like a prime minister", using the simile of rhetoric. Parables illustrate both have lung assist, assistant functions, but also be able to explain the ethics inherent in traditional Chinese medicine.

Improve the "Internal Classic. Plain Questions" Culture - Loaded Words Translation Effect Cultural complexity and diversity determines the choice of translation strategies to reflect the work and flexible. Because the purpose of translation is to promote communication and integration of different cultures, so the appropriate changes can effectively improve the English translation effect is particularly necessary.

Avoid De-contextualized Translation of Culture-Loaded Words. Traditional Chinese language has a strong ambiguity, grammatical relations embodied recessive [4]. The English language belong which has a more distinct grammatical relation. Meanwhile, Chinese pay attention to "savvy", which involves a lot of language implied meaning, situation arise, the meaning of the word because the context changes and change. It must clarify the source language based on context, and to the implications of grammatical relations, to determine its meaning in the article. Such as "yin and yang", the pathology, the sun is empirical, heat syndrome, prima facie evidence, the female is a deficiency, poor circulation, where permits; yin and yang of a plant can flourish and sparse, and the sun back, women and men, etc. . Another example is China's traditional culture of "Tao" is the "criteria approach" in the formation of philosophical concepts of Taoism of continuous improvement. Plain Questions", the channel has different meanings in different contexts and it can be expressed as ideas, doctrine, laws and methods.

Try To Choose Transliteration Plus Explanatory Increase Translation. Translation philosophy class, class names, you can select the translation mode when increase translation terminology class culture-loaded words. Chinese medicine is gradually formed over thousands of years of medical system, and modern Western medicine has significant differences, which carries a particularly profound Chinese culture and humanistic ideas. During the development of Chinese medicine by the ancient geography, ancient astronomy, ancient philosophy permeate and influence of knowledge, it does not take the premise and foundation of modern anatomy, but on the basis of observation and verification, and gradually improve and perfect the experience of medicine. Chinese medicine and Western medicine compared to its Humanities and more vivid colors, speculative philosophy of Chinese medicine is the main method of thinking, inquiry and treatment.

"Internal Classic. Plain Questions" appear frequently in ancient names, but difficult to find the corresponding English words. Translating "Yellow Emperor" of Chinese medicine in China and its purpose is to spread culture to other countries. To fully reflect the English translation of the original Chinese medicine and the corresponding lack of English vocabulary, transliteration plus explanation is an ideal choice. Chinese medicine is not only to retain the concept and content, but also make the translation consistent. Convey meaning, we should make more use of interpretative translations [5]. In accordance with their different languages and language habits of expression, in the translation of the sentence or phrase added. Purpose is to translate the source language that works well understood and the target language readers but feel awkward, surprised connotation, to facilitate understanding, to achieve the desired effect of translation, thus achieving cross-cultural communication.

Other Culture-Loaded Words is to be Translated Phonetically and to Give Readers A 
Better Understanding of the Article. Many culture-loaded words is not the focus of the term composed of traditional Chinese medicine theory, but in the "Q" describes a high medical knowledge and language of the position [6]. These words are fuzzy, synonyms and ambiguity, its flexible use, increase the difficulty of understanding the translator, requiring translation of ancient Chinese people have a higher capacity, and read a lot of literature, reference books, do a lot of Exegesis jobs. But some vocabulary words in English and there is a corresponding relationship, so the translation of these terms is to understand the focus, if the improper understanding will produce a lot of mistranslation. The translation of the priority is to help the reader understanding of the original meaning, so that the natural language of coherence. Meaning not only can reduce the use of the reader's understanding of the issues, but also reduce the "Translated" to improve translation fluency and readability.

Select the Comment form Depending on the Circumstances. Plain Questions" translation take different annotation method to increase the accuracy of the translation with rich content translation, it is worth learning and reference. First, he chose to translate the word in quotation marks mode to blur the words translate fuzzy words and can be arranged in the literal translation of the selected word. Because the Chinese language has a strong specificity, even if some of the terms of use of all translation methods can not articulate it has all the connotations. Note at this time should be chosen Chinese folder, for learners to read and provide convenient access to information.

\section{Conclusion}

Translation is part of cross-cultural communication, which includes the original language, the reader, the translator, the integration of aesthetics psychology, philosophy, culture and language. Translating "Internal Classic Plain Questions" Culture - loaded Words has a significant impact on the spread of Chinese culture, its smooth, wide dissemination of key areas. Load word translation in many ways often uses a literal translation, literal filling method, transliteration, transliteration filling method When translating "Q" to avoid de-contextualized translation of culture-loaded words; to select a transliteration plus explanatory increase translation; other culture-loaded words should to be translated phonetically to give readers a better understanding of the article and select the comment form depending on the circumstances effectively enhance the effect of TCM Classics into English.

\section{Acknowledgements}

Fund Project: Heilongjiang Province 2016 focused on economic and social development research (Western disciplines special) general topic "Culture - loaded Words Translation Strategy of TCM classics Skopos-theory Perspective -" Internal Classic. Plain Questions "Translation Studies.

Fund Number WY2016061 -B

\section{References}

[1] Wu Chunyu, Wang Yinquan. Ecological Perspective of Translation Studies "Yellow Emperor" Culture - loaded Words Translation [J]. Chinese Medicine, 2015,01: 61-64.

[2] Li Lei. in the medical literature in translation studies culture-loaded words under Schema Perspective [J]. Chinese Medicine, 2015,01: 107-110.

[3] Yang Liwen, Wang Yinquan. "Yellow Emperor" Translation Studies in China (2000-2014) [J]. Traditional Chinese Medicine, 2015, 12: 105-108 + 112.

[4] Li Shanshan. Translation of the "Yellow Emperor" culture-loaded words under Hermeneutic [J]. Modern communication, 2016,06: 65-66.

[5] Shi Weiping. On due in Vision "Yellow Emperor" Culture - specific Items Translation Strategies Shi Weiping mold [J]. Shanghai University of Traditional Chinese Medicine, 2014,05: 15-18.

[6] Zhou Dongmei. Adaptation of Translation "Yellow Emperor" Chinese Culture - loaded Words 
Perspective [J]. Zhejiang University of Traditional Chinese Medicine, 2012, 10: 1139-1141. 